\title{
Dual Entropy in Discrete Groups with Amenable Actions
}

\author{
Nathanial P. Brown 1 \\ UC-Berkeley \\ Berkeley, California 94720 \\ nbrown@math.berkeley.edu \\ Emmanuel Germain \\ Université de Paris VII \\ UFR de Mathématiques, 75005 Paris \\ germain@math.jussieu.fr
}

\begin{abstract}
Let $G$ be a discrete group which admits an amenable action on a compact space and $\gamma \in A u t(G)$ be an automorphism. We define a notion of entropy for $\gamma$ and denote the invariant by $h a(\gamma)$. This notion is dual to classical topological entropy in the sense that if $G$ is abelian then $h a(\gamma)=$ $h_{\text {Top }}(\hat{\gamma})$ where $h_{\text {Top }}(\hat{\gamma})$ denotes the topological entropy of the induced automorphism $\hat{\gamma}$ of the (compact, abelian) dual group $\hat{G}$.

$h a(\cdot)$ enjoys a number of basic properties which are useful for calculations. For example, it decreases in invariant subgroups and certain quotients. These basic properties are used to compute the dual entropy of an arbitrary automorphism of a crystallographic group.
\end{abstract}

\section{Introduction}

Let $\mathcal{G}$ denote the class of countable discrete groups which admit an amenable action on a compact space (cf. [ADR]; see also Definition 2.1 below). This notion has recently been very important in work related to the Novikov conjecture (cf. $[\mathrm{HR}]$ ). Recently it has also been shown that this class of groups coincides with the class of exact groups (cf. $[\mathrm{AD}],[\mathrm{GK}],[\mathrm{O}]) . \mathcal{G}$ is known to be very large; it contains the classical amenable groups, free groups (or any other hyperbolic group; $[\mathrm{Ad}],[\mathrm{Ge}]$ ) and discrete subgroups of connected Lie groups (cf. [Z]). Moreover, $\mathcal{G}$ is closed under a number of standard group operations such as taking subgroups, extensions, free products and quotients by classical amenable groups.

In this paper we study a conjugacy class invariant for automorphisms of elements in $\mathcal{G}$. The invariant, denoted $h a(\gamma)$ when $\gamma \in \operatorname{Aut}(G)$ and $G \in \mathcal{G}$, is called the dual entropy of $\gamma$. The terminology comes from the fact that if $G$ is abelian with (compact) dual group $\hat{G}$ then for each $\gamma \in \operatorname{Aut}(G), h a(\gamma)$ is equal to the classical topological entropy of the induced automorphism $\hat{\gamma} \in A u t(\hat{G})$.

1 NSF Postdoctoral Fellow.

1991 AMS Classification; 46L55. 
Our motivation for the present work is twofold. On the one hand, discrete groups are one of the most basic objects in mathematics and hence we feel it is worthwhile to have a numerical invariant for dynamical systems arising from such groups. In the framework of operator algebras, noncommutative dynamical systems have been studied for a long time. However, the various notions of entropy in this setting are still not well understood. Hence the second motivation for our work is to provide an invariant which is closely related to some of the operator algebra entropies and will hopefully provide a setting where hypotheses in general operator algebras can be more easily tested. For example, a result of Voiculescu concerning certain automorphisms of noncommutative tori will follow immediately from a general inequality relating dual entropy and noncommutative topological entropy as defined in [Br] (cf. Propositions 3.3, 3.5). Also, we will show that dual entropy decreases in quotients when the kernel is a classical amenable group (cf. Proposition 5.8). (The corresponding question for noncommutative topological entropy is still open.)

These notes are organized as follows. In section 2 we give the definition of dual entropy and show that there is always a canonical action which determines entropy calculations (Proposition 2.6). In section 3 we prove a general relationship between dual entropy and the noncommutative topological entropy defined in [Br] (cf. Proposition 3.3). In section 4 we justify the terminology "dual" entropy by treating the case of abelian groups. In section 5 we develop the basic properties of this entropy function. We will see that dual entropy satisfies all of the properties which are currently known for other noncommutative approximation entropies and has the added feature of decreasing in certain quotients. Finally in section 6 we give some calculations of dual entropy. In particular, we will give exact calculations of the dual entropy of an arbitrary automorphism of a crystallographic group (Corollary 6.7).

\section{Definition of Dual Entropy}

In this section, we define the dual entropy of an automorphism (or any other map) of a discrete group which admits an amenable action. Our definition is based on the approximation approach to noncommutative dynamical entropy which was first introduced in [Vo]. In [Vo] the main focus is operator algebras and the approximations involved deal with (various) norm approximations of operators on a Hilbert space. In the group context we replace norm approximations with approximate invariance and get a definition which behaves similar to those in $[\mathrm{Vo}]$ (see also $[\mathrm{Br}]$ ). In a recent preprint, M. Choda has independantly formulated a notion similar to our dual entropy in the setting of amenable groups (cf. [Ch]). It would be interesting to know whether or not these notions coincide for amenable groups. 
Before we can state the appropriate definitions, we must introduce some notation. If $G$ is a discrete group and $A$ is a unital commutative $C^{*}$-algebra we let $l^{1}(G, A)$ denote the closure of the linear space of finitely supported functions $T: G \rightarrow A$ with respect to the norm $\|T\|_{1}=\left\|\sum_{g}|T(g)|\right\|_{A}$.

Definition 2.1 Let $G$ be a discrete group and $\alpha: G \rightarrow A u t(A)$ be a homomorphism, where $A$ is a unital commutative $C^{*}$-algebra. Then the action $\alpha$ is called amenable if there exist functions $T_{n} \in l^{1}(G, A)$ such that $T_{n}$ is nonnegative (i.e. $T_{n}(g) \geq 0$ for all $g \in G$ ), finitely supported, $\sum_{g} T_{n}(g)=1_{A}$ and $\left\|s . T_{n}-T_{n}\right\|_{1} \rightarrow 0$ for all $s \in G$, where $s . T(g)=\alpha_{s}\left(T\left(s^{-1} g\right)\right)$ for all $T \in l^{1}(G, A)$.

It is clear that a group is amenable in the classical sense if and only if the trivial action $\tau: G \rightarrow A u t(\mathbb{C})$ is amenable in the sense described above. See also $[\mathrm{ADR}]$ for a comprehensive treatment of general amenable groupoids.

Definition 2.2 If $G$ is a discrete group, $\alpha: G \rightarrow A u t(A)$ is an amenable action, $\omega \subset G$ is a finite set and $\delta>0$ then put

$$
r a(\alpha, \omega, \delta)=\inf \left\{|\operatorname{supp}(T)|:\|s . T-T\|_{1}<\delta \text { for all } s \in \omega\right\}
$$

where the infimum is taken over all nonnegative functions $T \in l^{1}(G, A)$ with $\sum_{g} T(g)=1_{A}$ and $|\operatorname{supp}(T)|$ denotes the cardinality of the support of $T$. The integer $r a(\alpha, \omega, \delta)$ is called the amenable $\delta$-rank of $\omega$ with respect to $\alpha$. One then defines

$$
r a(\omega, \delta)=\inf _{\alpha} r a(\alpha, \omega, \delta),
$$

where the infimum is taken over all amenable actions $\alpha: G \rightarrow A u t(A)$ (and, of course, $A$ is allowed to vary). Then $r a(\omega, \delta)$ is called the amenable $\delta$-rank of $\omega$.

We will soon see that the infimum defining $r a(\omega, \delta)$ is always realized at a canonical action (Proposition 2.6). However, the present definition makes certain calculations easier.

Having a suitable " $\delta$-rank" function for finite sets we now mimic the definitions in $[\mathrm{Vo}]$ to get our definition of dual entropy.

Definition 2.3 If $G$ admits an amenable action and $\gamma \in A u t(G)$ we define the following quantities:

$$
\begin{gathered}
h a(\gamma, \omega, \delta)=\limsup _{n \rightarrow \infty} \frac{\log \left(r a\left(\omega \cup \gamma(\omega) \cup \ldots \cup \gamma^{n-1}(\omega), \delta\right)\right)}{n}, \\
h a(\gamma, \omega)=\sup _{\delta>0} h a(\gamma, \omega, \delta),
\end{gathered}
$$




$$
h a(\gamma)=\sup _{\omega} h a(\gamma, \omega),
$$

where the final supremum is taken over all finite subsets of $G$. We call $h a(\gamma)$ the dual entropy of $\gamma$.

Though stated for automorphisms, it is clear that the definitions above make perfectly good sense for endomorphisms (or any other self map) of $G$. Indeed, many of the results which are to follow have similar formulations and proofs in the case of endomorphisms.

Remark 2.4 The above definitions are also easily extended to cover arbitrary (locally compact) groups which admit amenable actions. Since Haar measure on a discrete group is just the counting measure, we could also write $\mu(\operatorname{supp}(T))$ in the definition of $r a(\alpha, \omega, \delta)$, where $\mu$ is Haar measure, and then it is clear how to extend this definition to cover more general groups. However, we will stick to the discrete case since this more general notion would have the philosophically unpleasant feature of always taking the value zero on any compact group.

Definition 2.5 If $\alpha: G \rightarrow \operatorname{Aut}(A)$ is an amenable action, $\omega \subset G$ is a finite set and $\delta>0$ then we say a funtion $T \in l^{1}(G, A)$ is minimal for $(\omega, \delta)$ if $T$ is nonnegative, $\sum_{g} T(g)=1_{A},\|s . T-T\|_{1}<\delta$ for all $s \in \omega$ and $|\operatorname{supp}(T)|=$ $r a(\alpha, \omega, \delta)$.

If $G$ is discrete we let $\alpha^{G}: G \rightarrow A u t\left(l^{\infty}(G)\right)$ denote the canonical action by left translation. That is, $\alpha_{g}^{G}(\zeta)=g . \zeta$ where $g . \zeta(x)=\zeta\left(g^{-1} x\right)$ for all $\zeta \in l^{\infty}(G)$. It is well known that $G$ admits an amenable action if and only if the action $\alpha^{G}$ is already amenable. However, it is an important fact that all entropy calculations are also determined by this canonical action.

Proposition 2.6 If $G$ admits an amenable action then for all finite subsets $\omega$ and $\delta>0, r a(\omega, \delta)=r a\left(\alpha^{G}, \omega, \delta\right)$. If $G$ is an amenable group then $\operatorname{ra}(\omega, \delta)=$ $\operatorname{ra}(\tau, \omega, \delta)$, where $\tau: G \rightarrow$ Aut $(\mathbb{C})$ is the trivial action.

Proof. Let $\omega \subset G$ be a finite set and $\delta>0$. Choose an amenable action $\alpha: G \rightarrow \operatorname{Aut}(C(X))$ for which $r a(\omega, \delta)=r a(\alpha, \omega, \delta)$ and take $T \in l^{1}(G, C(X))$ minimal for $(\omega, \delta)$ (Definition 2.5).

Fix some point $x_{0} \in X$ and consider the unital *-homomorphism $\Lambda: C(X) \rightarrow$ $l^{\infty}(G)$ given by $\xi \mapsto \Lambda(\xi)$ such that $\Lambda(\xi)(g)=\alpha_{g}(\xi)\left(x_{0}\right)$ for all $g \in G$ and $\xi \in C(X)$. The important observation is that $\Lambda$ intertwines the actions $\alpha$ and $\alpha^{G}$. 
Recall that we have $T \in l^{1}(G, C(X))$ minimal for $(\omega, \delta)$ and hence we define $S \in l^{1}\left(G, l^{\infty}(G)\right)$ by $S(g)=\Lambda(T(g))$. Then it is clear that $S$ is nonnegative, $\sum_{g} S(g)=1_{l \infty(G)}$ and $\operatorname{supp}(S) \subset \operatorname{supp}(T)$. Moreover, for each $h \in G$ we have

$$
\begin{aligned}
\|h . S-S\|_{1} & =\left\|\sum_{g \in G}\left|\alpha_{h}^{G}\left(\Lambda\left(T\left(h^{-1} g\right)\right)\right)-\Lambda(T(g))\right|\right\|_{l^{\infty}(G)} \\
& \left.=\| \Lambda\left(\sum_{g \in G} \mid \alpha_{h}\left(T\left(h^{-1} g\right)\right)-T(g)\right) \mid\right) \|_{l^{\infty}(G)} \\
& \left.\leq \| \sum_{g \in G} \mid \alpha_{h}\left(T\left(h^{-1} g\right)\right)-T(g)\right) \mid \|_{C(X)} \\
& =\|h . T-T\|_{1} .
\end{aligned}
$$

Thus we see that $r a\left(\alpha^{G}, \omega, \delta\right) \leq|\operatorname{supp}(S)| \leq|\operatorname{supp}(T)|=r a(\omega, \delta)$. However, the opposite inequality is immediate from the definition and so we have proved the first part of the proposition.

Assume now that $G$ is an amenable group and let $m$ be a left invariant mean on $l^{\infty}(G)$ (i.e. $m$ is a state on $l^{\infty}(G)$ with the property that $m\left(\alpha_{h}^{G}(\zeta)\right)=$ $m(h . \zeta)=m(\zeta)$ for all $\zeta \in l^{\infty}(G)$ and $\left.h \in G\right)$. Let $\tau: G \rightarrow \operatorname{Aut}(\mathbb{C})$ be the trivial action and by the first part of the proof we only have to show that $r a(\tau, \omega, \delta) \leq r a\left(\alpha^{G}, \omega, \delta\right)$ for all finite subsets $\omega \subset G$ and $\delta>0$. So choose $T \in l^{1}\left(G, l^{\infty}(G)\right)$ minimal for $(\omega, \delta)$. Then define $S \in l^{1}(G)=l^{1}(G, \mathbb{C})$ as $S(g)=m(T(g))$ and for all $h \in G$ we have

$$
\begin{aligned}
\|h . S-S\|_{1} & =\sum_{g \in G}\left|S\left(h^{-1} g\right)-S(g)\right| \\
& =\sum_{g \in G}\left|m\left(T\left(h^{-1} g\right)\right)-m(T(g))\right| \\
& =\sum_{g \in G}\left|m\left(\alpha_{h}^{G}\left(T\left(h^{-1} g\right)\right)\right)-m(T(g))\right| \\
& \leq m\left(\sum_{g \in G}\left|\alpha_{h}^{G}\left(T\left(h^{-1} g\right)\right)-T(g)\right|\right) \\
& \leq\|h . T-T\|_{1} .
\end{aligned}
$$

Since it is clear that $S$ is nonnegative, $\sum_{g} S(g)=1$ and $\operatorname{supp}(S) \subset \operatorname{supp}(T)$ (actually, we have equality by the minimality of $T$ and the estimates above) we see that $r a(\tau, \omega, \delta) \leq r a\left(\alpha^{G}, \omega, \delta\right)=r a(\omega, \delta)$. 


\section{Comparison with Noncommutative Topologi- cal Entropy}

In this section we show that dual entropy on a discrete group dominates the noncommutative topological entropy (as defined in $[\mathrm{Br}]$ ) on the reduced group $C^{*}$-algebra. In fact, the same inequality holds when the group has a $\mathbb{C}$ valued cocycle and hence we will immediately deduce a result of Voiculescu for certain natural automorphisms of noncommutative tori. See $[\mathrm{Br}]$ for all the definitions and notation related to noncommutative topological entropy.

If $\alpha: G \rightarrow \operatorname{Aut}(A)$ in an action on a unital commutative $C^{*}$-algebra and $S, T: G \rightarrow A$ are finitely supported functions we define the $\alpha$-convolution as follows:

$$
S *_{\alpha} T(h)=\sum_{g \in G} S(g) \alpha_{g}\left(T\left(g^{-1} h\right)\right) .
$$

We also define the function $T^{*}$ by $T^{*}(h)=\alpha_{h}\left(T\left(h^{-1}\right)^{*}\right)$. Let $l^{2}(G, A)$ denote the closure of the finitely supported functions from $G$ to $A$ with respect to the norm $\|T\|_{2}=\left\|\sum_{g} T(g)^{*} T(g)\right\|_{A}^{1 / 2} . l^{2}(G, A)$ is a Hilbert $A$-module with respect to the inner product $\langle T, S\rangle=\sum_{g} T(g)^{*} S(g)$ and we have the usual Cauchy-Schwartz inequality $\|<T, S>\|_{A} \leq\|T\|_{2}\|S\|_{2}$.

Lemma 3.1 If $T: G \rightarrow A$ is finitely supported, nonnegative, $\sum_{g} T(g)=1_{A}$ and $S: G \rightarrow A$ is defined by $S(g)=T(g)^{1 / 2}$, then for all $h \in G$ we have $\left\|1_{A}-S *_{\alpha} S^{*}(h)\right\|_{A}^{2} \leq\|h . T-T\|_{l^{1}(G, A)}$.

Proof. The proof is a calculation analogous to that in [Pe, 7.3.8] only a bit more care must be taken since we are dealing with modules and functions.

$$
\begin{aligned}
\left\|1_{A}-S *_{\alpha} S^{*}(h)\right\|_{A}^{2} & =\|<S, S-h \cdot S>\|_{A}^{2} \\
& \leq\|S-h \cdot S\|_{2}^{2} \\
& =\left\|\sum_{g \in G}\left|S(g)-\alpha_{h}\left(S\left(h^{-1} g\right)\right)\right|^{2}\right\|_{A} \\
& \leq\left\|\sum_{g \in G}\left|S(g)^{2}-\alpha_{h}\left(S\left(h^{-1} g\right)\right)^{2}\right|\right\|_{A} \\
& =\|T-h \cdot T\|_{1} .
\end{aligned}
$$

Definition 3.2 A map $\theta: G \times G \rightarrow \mathbb{T}=\{c \in \mathbb{C}:|c|=1\}$ is called a $\mathbb{C}$-cocycle if $\theta(1, g)=\theta(g, 1)=1$, for all $g \in G$ and $\theta(g, h) \theta(g h, k)=\theta(h, k) \theta(g, h k)$ for all $g, h, k \in G$. 
If $\alpha: G \rightarrow \operatorname{Aut}(A)$ is an action where $A \subset B(H)$ then we define a *monomorphism $\pi: A \rightarrow B\left(l^{2}(G) \otimes H\right)$ by $\pi(a) \xi_{g} \otimes h=\xi_{g} \otimes \alpha_{g^{-1}}(a) h$ for all $\xi_{g} \in l^{2}(G)$ and $h \in H$. ( $\left\{\xi_{g}\right\}_{g \in G}$ denotes the canonical orthonormal basis.) We also define a unitary representation $\lambda^{\theta}: G \rightarrow B\left(l^{2}(G) \otimes H\right)$ by $\lambda_{s}^{\theta}\left(\xi_{g} \otimes h\right)=$ $\theta(s, g) \xi_{s g} \otimes h$. Then the reduced cocycle crossed product $A \rtimes_{r, \alpha}(G, \theta)$ is defined to be the $C^{*}$-algebra generated by $\left\{\pi(a) \lambda_{s}^{\theta}: a \in A, s \in G\right\}$. One has the relations $\lambda_{s}^{\theta} \lambda_{t}^{\theta}=\theta(s, t) \lambda_{s t}^{\theta}$ and $\lambda_{s}^{\theta} \pi(a) \lambda_{s}^{\theta *}=\pi\left(\alpha_{s}(a)\right)$. When $A=\mathbb{C}$ we use the notation $C_{r}^{*}(G, \theta)$. Note that if $\gamma \in \operatorname{Aut}(G)$ and satisfies the relation $\theta(g, h)=$ $\theta(\gamma(g), \gamma(h))$ for all $g, h \in G$ then there is a natural induced automorphism $\hat{\gamma} \in \operatorname{Aut}\left(C_{r}^{*}(G, \theta)\right)$ defined by $\hat{\gamma}\left(\lambda_{g}^{\theta}\right)=\lambda_{\gamma(g)}^{\theta}$.

Proposition 3.3 If $\theta: G \times G \rightarrow \mathbb{C}$ is a $\mathbb{C}$-cocycle and $\gamma \in \operatorname{Aut}(G)$ is such that $\theta(g, h)=\theta(\gamma(g), \gamma(h))$ for all $g, h \in G$ then $h t(\hat{\gamma}) \leq h a(\gamma)$.

Proof. The proof is an adaptation of the techniques in [SS], [Br] and [BC]: thus we will be rather sketchy. Let $\alpha^{G}: G \rightarrow \operatorname{Aut}\left(l^{\infty}(G)\right)$ be the canonical action and construct $l^{\infty}(G) \rtimes_{r, \alpha^{G}}(G, \theta)$ as above.

By the Kolmogorov-Sinai type result ([Br, Prop. 2.6]) and Proposition 2.6 it suffices to show that if $\omega \subset G$ is a finite set then $\operatorname{rcp}\left(\iota, \lambda^{\theta}(\omega), \delta^{1 / 2}\right) \leq r a\left(\alpha^{G}, \omega, \delta\right)$ for all $\delta>0$, where $\iota: l^{\infty}(G) \rtimes_{r, \alpha^{G}}(G, \theta) \hookrightarrow B\left(l^{2}(G) \otimes H\right)$ is the natural inclusion. So, choose $T \in l^{1}\left(G, l^{\infty}(G)\right)$ which is minimal for $(\omega, \delta)$ and let $F=\operatorname{supp}(T) \subset G$. Let $P_{F} \in B\left(l^{2}(G)\right)$ be the orthogonal projection onto the span of $\left\{\xi_{h}: h \in F\right\}$. Let $X=\sum_{p \in F} e_{p, p} \otimes \alpha_{p^{-1}}^{G}(S(p)) \in M_{|F|} \otimes l^{\infty}(G)$, where $S(p)=T(p)^{1 / 2}$ and $e_{p, q}$ denote the canonical matrix units of $M_{|F|}=$ $P_{F} B\left(l^{2}(G)\right) P_{F}$. Then one computes

$X\left(P_{F} \otimes I\right) \lambda_{h}^{\theta}\left(P_{F} \otimes I\right) X=\sum_{p \in F \cap h F} \theta\left(h, h^{-1} p\right) e_{p, h^{-1} p} \otimes \alpha_{p^{-1}}^{G}(S(p)) \alpha_{p^{-1} h}^{G}\left(S\left(h^{-1} p\right)\right)$.

Now define the completely positive map $L: M_{|F|} \otimes l^{\infty}(G) \rightarrow l^{\infty}(G) \rtimes_{r, \alpha^{G}}$ $(G, \theta)$ by $L\left(e_{x, y} \otimes \zeta\right)=\overline{\theta\left(x y^{-1}, y\right)} \pi\left(\alpha_{x}^{G}(\zeta)\right) \lambda_{x y^{-1}}^{\theta}$. Then a straightforward (but somewhat unpleasant) calculation shows that

$$
L\left(X\left(P_{F} \otimes I\right) \lambda_{h}^{\theta}\left(P_{F} \otimes I\right) X\right)=\pi\left(S *_{\alpha^{G}} S^{*}(h)\right) \lambda_{h}^{\theta},
$$

for all $h \in G$. Hence $\left\|\lambda_{h}^{\theta}-L\left(X\left(P_{F} \otimes I\right) \lambda_{h}^{\theta}\left(P_{F} \otimes I\right) X\right)\right\| \leq \delta^{1 / 2}$ for all $h \in \omega$, by Lemma 3.1. But since the map $\lambda_{h}^{\theta} \mapsto L\left(X\left(P_{F} \otimes I\right) \lambda_{h}^{\theta}\left(P_{F} \otimes I\right) X\right)$ factors through the matrix algebra $M_{|F|}$ this implies the desired inequality.

Remark 3.4 It follows from [Dy, Prop. 9] and the proposition above that the dual entropy also dominates CNT-entropy (cf. [CNT]) with respect to the canonical trace on $C_{r}^{*}(G)$ (and in the $W^{*}$-algebra $L(G)$ ). Also, the proof above together with the results of [BC, Section 2] can be used to show that dual entropy 
dominates the $W^{*}$-entropy defined in [Vo, Section 3] in the case that $G$ is an amenable group.

Let $\theta: \mathbb{Z}^{n} \times \mathbb{Z}^{n} \rightarrow \mathbb{T}$ be a $\mathbb{C}$-cocycle. Then $C_{r}^{*}\left(\mathbb{Z}^{n}, \theta\right)$ is the usual noncommutative torus with generating unitaries $\lambda_{1}^{\theta}, \ldots, \lambda_{n}^{\theta}\left(\lambda_{i}^{\theta}=\lambda_{e_{i}}^{\theta}\right.$ for the canonical generators $\left\{e_{i}\right\}_{1 \leq i \leq n}$ of $\left.\mathbb{Z}^{n}\right)$ subject to the relations $\lambda_{i}^{\theta} \lambda_{j}^{\theta}=\theta\left(e_{i}, e_{j}\right) \lambda_{i+j}^{\theta}=$ $\theta\left(e_{i}, e_{j}\right) \overline{\theta\left(e_{j}, e_{i}\right)} \lambda_{j}^{\theta} \lambda_{i}^{\theta}$.

Corollary 3.5 (Vo, Prop. 5.3) If $\gamma \in G L\left(n, \mathbb{Z}^{n}\right)=\operatorname{Aut}\left(\mathbb{Z}^{n}\right)$ is a matrix such that $\theta(\gamma(g), \gamma(h))=\theta(g, h)$ for all $g, h \in \mathbb{Z}^{n}$ then $h t(\hat{\gamma}) \leq h a(\gamma)=$ $\log \left(\mu_{1} \mu_{2} \cdots \mu_{n}\right)$, where $\hat{\gamma}$ is the induced automorphism of the noncommutative torus $C_{r}^{*}\left(\mathbb{Z}^{n}, \theta\right)$ and $\mu_{j}=\max \left(1,\left|t_{j}\right|\right), 1 \leq j \leq n$, for the eigenvalues $t_{j}$ of $\gamma$.

Proof. In the next section we will show that $h a(\gamma)=\log \left(\mu_{1} \mu_{2} \cdots \mu_{n}\right)$, since the dual group of $\mathbb{Z}^{n}$ is the (commutative) $n$-torus and it is well known how to compute the topological entropy of automorphisms of $\mathbb{T}^{n}$. Hence the corollary follows from Proposition 3.3.

\section{The Abelian Case}

Our next goal is to justify the terminology "dual" entropy. We will need a theorem of J. Peters (see [Pet, Thm. 6]).

Theorem 4.1 Let $G$ be a discrete abelian group with dual group $\hat{G}$. If $\gamma \in$ Aut $(G)$ with induced automorphism $\hat{\gamma} \in A u t(\hat{G})$ then the (classical) topological entropy, $h_{\text {Top }}(\hat{\gamma})$, of $\hat{\gamma}$ is equal to

$$
\sup _{E}\left(\limsup _{n \rightarrow \infty} \frac{\log \left|E+\gamma(E)+\ldots+\gamma^{n-1}(E)\right|}{n}\right)
$$

where the supremum is taken over all finite subsets $E \subset G$.

Theorem 4.2 If $G$ is discrete and abelian, $\gamma \in \operatorname{Aut}(G)$ and $\hat{\gamma} \in \operatorname{Aut}(\hat{G})$ is the induced automorphism then $h a(\gamma)=h_{\text {Top }}(\hat{\gamma})$.

Proof. By Proposition 3.3 above, [Br, Prop. 1.4] and [Vo, Prop. 4.8] we have the inequality $h a(\gamma) \geq h_{T o p}(\hat{\gamma})$. To prove the other inequality we apply Proposition 2.6 (recall that abelian groups are amenable) and Peters' theorem above. One basic fact we will need is that if $f_{1}, f_{2} \in l^{1}(G)$ are nonnegative functions then $\left\|f_{1} * f_{2}\right\|_{1}=\left\|f_{1}\right\|_{1}\left\|f_{2}\right\|_{1}$ and $\operatorname{supp}\left(f_{1} * f_{2}\right)=\operatorname{supp}\left(f_{1}\right)+\operatorname{supp}\left(f_{2}\right)$. 
So let $\omega \subset G$ be a finite set and $\delta>0$. Choose some nonnegative, norm one function of finite support $f \in l^{1}(G)$ such that $\|s . f-f\|<\delta$ for all $s \in \omega$. Now define $F_{n}=f * f \circ \gamma^{-1} * \cdots * f \circ \gamma^{-n+1}$. Then one verifies (using that convolution is commutative since $G$ is abelian) that $\left\|s . F_{n}-F_{n}\right\|<\delta$ for all $s \in \omega \cup \cdots \cup \gamma^{n-1}(\omega)$. Hence $r a\left(\omega \cup \cdots \cup \gamma^{n-1}(\omega), \delta\right) \leq\left|\operatorname{supp}\left(F_{n}\right)\right|$. But $\operatorname{supp}\left(F_{n}\right)=\operatorname{supp}(f)+\cdots+\gamma^{n-1}(\operatorname{supp}(f))$ and hence one deduces that $h a(\gamma, \omega, \delta)$ is less than or equal to Peters' formula and hence is bounded above by $h_{\text {Top }}(\hat{\gamma})$.

\section{Basic Properties}

In this section we develop the basic properties of the fuction $h a(\cdot)$. Some of the proofs run parallel to the corresponding properties for the approximation entropies defined in $[\mathrm{Vo}]$ and hence we will often refer to that paper for details.

We begin by observing that $r a(\cdot, \cdot)$ is an isomorphism invariant.

Lemma 5.1 Let $\Phi: G \rightarrow H$ be a group isomorphism. For all finite subsets $\omega \subset G$ and $\delta>0$ we have $r a(\omega, \delta)=\operatorname{ra}(\Phi(\omega), \delta)$.

Proof. Let $\alpha: G \rightarrow A u t(A)$ be an amenable action and take $T \in l^{1}(G, A)$ minimal for $(\omega, \delta)$ (cf. Definition 2.5). Define an action $\beta: H \rightarrow \operatorname{Aut}(A)$ by $\beta(h)=\alpha_{\Phi^{-1}(h)}$ and $S \in l^{1}(H, A)$ by $S(h)=T\left(\Phi^{-1}(h)\right)$. Then one checks that $\|h . S-S\|_{1}=\|g . T-T\|_{1}$ for all $h=\Phi(g) \in H$. This evidently implies $\operatorname{ra}(\omega, \delta) \geq \operatorname{ra}(\Phi(\omega), \delta)$. The opposite inequality is similar.

Proposition 5.2 For all $\gamma, \sigma \in \operatorname{Aut}(G)$, ha $(\gamma)=h a\left(\sigma \circ \gamma \circ \sigma^{-1}\right)$.

Proof. This is an easy consequence of the previous lemma.

Proposition 5.3 For all $\gamma \in \operatorname{Aut}(G)$ and $k \in \mathbb{Z}$ we have $h a\left(\gamma^{k}\right)=|k| h a(\gamma)$.

Proof. With Lemma 5.1 in hand, the proof of this is quite similar to the proof of [Vo, Prop. 1.3] as it depends only on the algebraic part of the definition of $h a(\cdot)$ and not on the particular $\delta$-rank function being used.

Proposition 5.4 If $\omega_{1} \subset \omega_{2} \subset \ldots$ are finite sets with the property that

$$
G=\bigcup_{i \in \mathbb{N}, n \in \mathbb{Z}} \gamma^{n}\left(\omega_{i}\right)
$$

then $h a(\gamma)=\sup _{i \in \mathbb{N}} h a\left(\gamma, \omega_{i}\right)$. 
Proof. This is similar to the proof of [Vo, Prop. 3.4]. However, the reader will likely find it easier to give a proof independantly as one has no topological considerations in this setting.

Proposition 5.5 (Monotonicity) If $\gamma \in A u t(G)$ and $H \subset G$ is a subgroup such that $\gamma(H)=H$ then $h a\left(\left.\gamma\right|_{H}\right) \leq h a(\gamma)$.

Proof. Let $A$ be abelian, $\alpha: G \rightarrow A u t(A)$ be an amenable action and $\beta: H \rightarrow$ $\operatorname{Aut}(A)$ denote the restriction of $\alpha$ to $H$. It suffices to show that for all finite subsets $\omega \subset H$ and $\delta>0, r a(\beta, \omega, \delta) \leq \operatorname{ra}(\alpha, \omega, \delta)$.

Let $G / H$ be the space of right cosets and choose representatives $\left\{p_{i}\right\}_{i \in G / H} \subset$ $G$. Then each element of $G$ has a unique representation as $h p_{j}$ for some $h \in H$ and $j \in G / H$. Now choose $T \in l^{1}(G, A)$ which is minimal for $(\omega, \delta)$ and define $S \in l^{1}(H, A)$ by

$$
S(h)=\sum_{i \in G / H} T\left(h p_{i}\right) .
$$

Since $T$ is finitely supported, the summation above is finite. It is clear that $S$ is nonnegative, $\sum_{h \in H} S(h)=\sum_{h} \sum_{i} T\left(h p_{i}\right)=1_{A}$ and $|\operatorname{supp}(S)| \leq|\operatorname{supp}(T)|$. Moreover, for all $h \in H$,

$$
\begin{aligned}
\|h . S-S\|_{1} & =\left\|\sum_{k \in H}\left|\beta_{h}\left(S\left(h^{-1} k\right)\right)-S(k)\right|\right\| \\
& =\left\|\sum_{k \in H}\left|\sum_{i \in G / H} \alpha_{h}\left(T\left(h^{-1} k p_{i}\right)\right)-T\left(k p_{i}\right)\right|\right\| \\
& \leq\left\|\sum_{g \in G}\left|\alpha_{h}\left(T\left(h^{-1} g\right)\right)-T(g)\right|\right\| \\
& =\|h . T-T\|_{1} .
\end{aligned}
$$

This implies $r a(\beta, \omega, \delta) \leq r a(\alpha, \omega, \delta)$.

We now turn to the question of how $h a(\cdot)$ behaves in quotients. We rather doubt that the dual entropy always decreases but it does behave well when the kernel is amenable. However, this will require some preliminary results.

So assume that $1 \rightarrow K \rightarrow G \stackrel{\pi}{\rightarrow} H \rightarrow 1$ is an exact sequence where $K$ is an amenable group with left invariant mean $m \in S\left(l^{\infty}(K)\right)$. Let $\varphi: H \rightarrow G$ be a unital (set theoretic) splitting (i.e. $\varphi\left(1_{H}\right)=1_{G}$ and $\pi(\varphi(h))=h$ for all $h \in H$ ) and $\theta: H \times H \rightarrow K$ the corresponding cocycle map (i.e. $\varphi\left(h_{1}\right) \varphi\left(h_{2}\right)=$ $\varphi\left(h_{1} h_{2}\right) \theta\left(h_{1}, h_{2}\right)$ for all $\left.h_{1}, h_{2} \in H\right)$. For each $\zeta \in l^{\infty}(G)$ and $h \in H$, let $\zeta(\varphi(h) \cdot) \in l^{\infty}(K)$ denote the function $k \mapsto \zeta(\varphi(h) k)$. Define a linear map $\Lambda: l^{\infty}(G) \rightarrow l^{\infty}(G)$ by

$$
\Lambda(\zeta)(\varphi(h) k)=m(\zeta(\varphi(h) \cdot))
$$


for all $g=\varphi(h) k \in G$.

Lemma $5.6 \Lambda: l^{\infty}(G) \rightarrow l^{\infty}(G)$ is independant of the splitting $\varphi$. Also, for all $s \in G$ and $\zeta \in l^{\infty}(G), \Lambda\left(\alpha_{s}^{G}(\zeta)\right)=\alpha_{s}^{G}(\Lambda(\zeta))$.

Proof. Let $\psi: H \rightarrow G$ be any other unital splitting. Then for each $h \in H$ there exists $k_{h} \in K$ such that $\psi(h)=\varphi(h) k_{h}$. So for $\zeta \in l^{\infty}(G)$ we have $\zeta(\psi(h) \cdot)(k)=$ $\zeta\left(\varphi(h) k_{h} k\right)=\alpha_{k_{h}^{-1}}^{K}(\zeta(\varphi(h) \cdot))(k)$. Hence $m(\zeta(\psi(h) \cdot))=m(\zeta(\varphi(h) \cdot))$ and we see that $\Lambda$ does not depend on $\varphi$.

Now let $s=p \varphi(q)^{-1} \in G$ be arbitrary. For each $\zeta \in l^{\infty}(G), x \in H$ and $k \in K$ we have

$$
\begin{aligned}
\alpha_{s}^{G}(\zeta)(\varphi(x) \cdot)(k) & =\alpha_{s}^{G}(\zeta)(\varphi(x) k) \\
& =\zeta\left(\varphi(q) p^{-1} \varphi(x) k\right) \\
& =\zeta\left(\varphi(q x) \theta(q, x) \varphi(x)^{-1} p^{-1} \varphi(x) k\right) \\
& =\alpha_{t^{-1}}^{K}(\zeta(\varphi(q x) \cdot))(k),
\end{aligned}
$$

where $t=\theta(q, x) \varphi(x)^{-1} p^{-1} \varphi(x)$ (note that $\varphi(x)^{-1} p^{-1} \varphi(x) \in K$ by normality). Hence $m\left(\alpha_{s}^{G}(\zeta)(\varphi(x) \cdot)\right)=m(\zeta(\varphi(q x) \cdot))$. This implies that $\Lambda\left(\alpha_{s}^{G}(\zeta)\right)(\varphi(x) y)=$ $m(\zeta(\varphi(q x) \cdot))$.

A similar sort of argument shows $\alpha_{s}^{G}(\Lambda(\zeta))(\varphi(x) y)=m(\zeta(\varphi(q x) \cdot))$ and hence $\Lambda\left(\alpha_{s}^{G}(\zeta)\right)=\alpha_{s}^{G}(\Lambda(\zeta))$ as claimed.

Lemma 5.7 If $\alpha^{G}: G \rightarrow A u t\left(l^{\infty}(G)\right)$ is amenable and $T \in l^{1}\left(G, l^{\infty}(G)\right)$ is minimal for $(\omega, \delta)$ then $\Lambda(T) \in l^{1}\left(G, l^{\infty}(G)\right)$ is also minimal for $(\omega, \delta)$ where $\Lambda(T)(g)=\Lambda(T(g))$.

Proof. Evidently $\Lambda$ is a positive linear map (i.e. if $\zeta \geq 0$ then $\Lambda(\zeta) \geq 0$ ). Hence if $\zeta \in l^{\infty}(G)$ takes values in $\mathbb{R}$ then $|\Lambda(\zeta)| \leq \Lambda(|\zeta|)$ since $\zeta \leq|\zeta|$. With this observation and the above fact that $\Lambda\left(\alpha_{s}^{G}(\zeta)\right)=\alpha_{s}^{G}(\Lambda(\zeta))$ for all $\zeta \in l^{\infty}(G)$, a routine calculation shows that $\|g \cdot \Lambda(T)-\Lambda(T)\|_{1} \leq\|g \cdot T-T\|_{1}$ for all $g \in G$. This implies the lemma since it is clear that $\Lambda(T)$ is nonnegative, $\operatorname{supp}(\Lambda(T)) \subset$ $\operatorname{supp}(T)$ (actually $=$ by the minimality of $T$ ) and $\sum_{g} \Lambda(T)(g)=1_{A}$.

Proposition 5.8 Assume $1 \rightarrow K \rightarrow G \stackrel{\pi}{\rightarrow} H \rightarrow 1$ is an exact sequence, where $K$ is an amenable group, and assume $\gamma \in \operatorname{Aut}(G)$ leaves $K$ invariant (i.e. $\gamma(K)=K)$. If $\dot{\gamma} \in \operatorname{Aut}(G / K)$ denotes the induced automorphism then $h a(\gamma) \geq$ $h a(\dot{\gamma})$. 
Proof. It suffices to show that for each finite subset $\omega \subset G$ and $\delta>0, \operatorname{ra}(\omega, \delta) \geq$ $\operatorname{ra}(\pi(\omega), \delta)$.

Let $\varphi: H \rightarrow G$ be a unital splitting with cocycle map $\theta: H \times H \rightarrow K$ and construct $\Lambda: l^{\infty}(G) \rightarrow l^{\infty}(G)$ as above. Let $\pi_{*}: l^{\infty}(H) \hookrightarrow l^{\infty}(G)$ denote the unital *-monomorphism induced by $\pi: G \rightarrow H$. One readily verifies that $\pi_{*}\left(\alpha_{\pi(x)}^{H}(\zeta)\right)=\alpha_{x}^{G}\left(\pi_{*}(\zeta)\right)$ for all $x \in G, \zeta \in l^{\infty}(H)$. Note also that $\pi_{*}\left(l^{\infty}(H)\right)=$ $\Lambda\left(l^{\infty}(G)\right)$ as subsets of $l^{\infty}(G)$.

Now choose $T \in l^{1}\left(G, l^{\infty}(G)\right)$ minimal for $(\omega, \delta)$. By Lemma 5.7 above we may assume that $T(g) \in \Lambda\left(l^{\infty}(G)\right)=\pi_{*}\left(l^{\infty}(H)\right)$ for all $g \in G$. Now define $S \in l^{1}\left(H, l^{\infty}(H)\right)$ by

$$
S(\pi(x))=\sum_{k \in K} \pi_{*}^{-1}(T(x k))
$$

for all $h=\pi(x) \in H$. Note that $S$ is nonnegative, $\sum_{h} S(h)=1_{l^{\infty}(H)}$ and $|\operatorname{supp}(S)| \leq|\operatorname{supp}(T)|$. Finally we compute

$$
\begin{aligned}
\|\pi(x) . S-S\|_{1} & =\left\|\sum_{h \in H}\left|\alpha_{\pi(x)}^{H}\left(S\left(\pi(x)^{-1} h\right)\right)-S(h)\right|\right\|_{l^{\infty}(H)} \\
& =\left\|\sum_{h \in H}\left|\sum_{k \in K} \pi_{*}^{-1}\left(\alpha_{x}^{G}\left(T\left(x^{-1} \varphi(h) k\right)\right)-T(\varphi(h) k)\right)\right|\right\|_{l^{\infty}(H)} \\
& =\left\|\sum_{h \in H}\left|\sum_{k \in K} \alpha_{x}^{G}\left(T\left(x^{-1} \varphi(h) k\right)\right)-T\left(x^{-1} \varphi(h) k\right)\right|\right\|_{l^{\infty}(G)} \\
& \leq\left\|\sum_{g \in G}\left|\alpha_{x}^{G}\left(T\left(x^{-1} g\right)\right)-T\left(x^{-1} g\right)\right|\right\|_{l^{\infty}(G)} \\
& =\|x . T-T\|_{1} .
\end{aligned}
$$

This together with Proposition 2.6 implies that $r a(\omega, \delta) \geq r a(\pi(\omega), \delta)$.

The next lemma is known to the experts so we only sketch the proof.

Lemma 5.9 If $H \subset G$ is a subgroup which admits an amenable action, $\omega \subset H$ is a finite set and $\delta>0$ then there exists $S \in l^{1}\left(G, l^{\infty}(G)\right)$ such that $S$ is nonnegative, has finite support, $\sum_{g} S(g)=1_{l^{\infty}(G)}$ and $\|h . S-S\|<\delta$ (w.r.t. the canonical action $\alpha^{G}$ ) for all $h \in \omega$.

Proof. As in Proposition 5.5 we write $G=\bigcup_{k \in H, i \in G / H} k p_{i}$ where $G / H$ denotes the space of right cosets. Define a unital *-monomorphism $\Lambda: l^{\infty}(H) \rightarrow l^{\infty}(G)$ by $\Lambda(\zeta)\left(k p_{i}\right)=\zeta(k)$. A routine calculation shows that for all $h \in H, \alpha_{h}^{G}(\Lambda(\zeta))=$ $\Lambda\left(\alpha_{h}^{H}(\zeta)\right)$. Taking $T \in l^{1}\left(H, l^{\infty}(H)\right)$ minimal for $(\omega, \delta)$ and defining $S(g)=$ 
$\Lambda(T(g))$ if $g \in H$ and $S(g)=0$ otherwise, one checks that $S \in l^{1}\left(G, l^{\infty}(G)\right)$ has the desired properties.

Let $G_{1} \stackrel{\varphi_{1}}{\rightarrow} G_{2} \stackrel{\varphi_{2}}{\rightarrow} G_{3} \stackrel{\varphi_{3}}{\rightarrow} \ldots$ be an inductive system of groups and assume that there exist automorphisms $\gamma_{i} \in \operatorname{Aut}\left(G_{i}\right)$ such that $\gamma_{i+1} \circ \varphi_{i}=\varphi_{i} \circ \gamma_{i}$ for all $i \in \mathbb{N}$. If $G=\underline{\lim } G_{i}$ is the inductive limit then there is a natural inductive limit automorphism $\gamma \in \operatorname{Aut}(G)$ with the property that $\gamma \circ \Phi_{i}=\Phi_{i} \circ \gamma_{i}$ for all $i \in \mathbb{N}$, where $\Phi_{i}: G_{i} \rightarrow G$ are the natural homomorphisms. Let $K_{i}=\operatorname{ker}\left(\Phi_{i}\right) \subset G_{i}$ and $\dot{\gamma}_{i}$ denote the induced automorphism of $G_{i} / K_{i}$ for all $i \in \mathbb{N}$.

Proposition 5.10 Let $G$ be an inductive limit of groups admitting amenable actions and $\gamma \in \operatorname{Aut}(G)$ be an inductive limit automorphism as above. If all of the subgroups $K_{i}$ are amenable (e.g. if the $\varphi_{i}$ are injective) then

$$
h a(\gamma)=\lim _{i \rightarrow \infty} h a\left(\dot{\gamma}_{i}\right) \leq \liminf _{i \rightarrow \infty} h a\left(\gamma_{i}\right) .
$$

Proof. Since Proposition 5.8 implies that the groups $G_{i} / K_{i}$ admit amenable actions, it follows from Lemma 5.9 that $G$ also admits an amenable action. Hence the proposition follows from Propositions 5.4, 5.5 and 5.8.

We next investigate how $h a(\cdot)$ behaves in extensions. Any general results in this direction appear to be very hard as it is not clear how to construct good actions on an extension when one is given actions on the kernel and quotient. The following lemma illustrates the difficulties. Our proof is a modification of an argument shown to us by Jean Renault and we thank him for sharing his notes with us.

As before, let $1 \rightarrow K \rightarrow G \stackrel{\pi}{\rightarrow} H \rightarrow 1$ be exact and let $\varphi: H \rightarrow G$ be a unital splitting with cocyle map $\theta: H \times H \rightarrow K$.

Lemma 5.11 Let $G$ be as above and assume both $K$ and $H$ admit amenable actions. Let $\omega=\left\{\left(\varphi\left(h_{1}\right) k_{1}\right)^{-1}, \ldots,\left(\varphi\left(h_{m}\right) k_{m}\right)^{-1}\right\} \subset G$ be a finite set and choose $T \in l^{1}\left(H, l^{\infty}(H)\right)$ minimal for $(\pi(\omega), \delta)$. If $F=\operatorname{supp}(T) \subset H$ then

$$
r a(\omega, 2 \delta) \leq r a(\pi(\omega), \delta) r a\left(\bigcup_{i=1}^{m} \bigcup_{x \in h_{i}^{-1} F} \varphi(x)^{-1} k_{i}^{-1} \varphi(x) \theta\left(h_{i}, x\right)^{-1}, \delta\right) .
$$

Proof. Choose $S \in l^{1}\left(K, l^{\infty}(K)\right)$ minimal for

$$
\left(\bigcup_{i=1}^{m} \bigcup_{x \in h_{i}^{-1} F} \varphi(x)^{-1} k_{i}^{-1} \varphi(x) \theta\left(h_{i}, x\right)^{-1}, \delta\right) .
$$

Now define $P: G \rightarrow l^{\infty}(G)$ by

$$
P(\varphi(x) y)(\varphi(q) p)=T(x)(q) S(y)\left(\varphi(x)^{-1} \varphi(q) p \varphi\left(q^{-1} x\right)\right) .
$$


Note that $\varphi(x)^{-1} \varphi(q) p \varphi\left(q^{-1} x\right)=\left(\varphi(x)^{-1}\left(\varphi(q) p \varphi(q)^{-1}\right) \varphi(x)\right) \theta\left(q, q^{-1} x\right) \in K$, by normality of $K$, and hence $P$ is well defined. It is clear that $P$ is nonnegative and finitely supported with $|\operatorname{supp}(P)| \leq|\operatorname{supp}(T)||\operatorname{supp}(S)|$. Moreover, for all $\varphi(q) p \in G$,

$$
\begin{aligned}
\sum_{\varphi(x) y \in G} P(\varphi(x) y)(\varphi(q) p) & =\sum_{\varphi(x) y \in G} T(x)(q) S(y)\left(\varphi(x)^{-1} \varphi(q) p \varphi\left(q^{-1} x\right)\right) \\
& =\sum_{x \in H} T(x)(q)\left(\sum_{y \in K} S(y)\left(\varphi(x)^{-1} \varphi(q) p \varphi\left(q^{-1} x\right)\right)\right) \\
& =\sum_{x \in H} T(x)(q)=1 .
\end{aligned}
$$

Hence $\sum_{g} P(g)=1_{l \infty(G)}$.

It remains to show the inequality $\|s . P-P\|_{1}<2 \delta$ for all $s=(\varphi(h) k)^{-1} \in \omega$. For notational reasons it will be convenient to define $A(x)=\theta(h, x) \varphi(x)^{-1} k \varphi(x)$ for all $x \in H$.

$$
\begin{aligned}
&\|s . P-P\|_{1}=\left\|\sum_{\varphi(x) y \in G}\left|\alpha_{s}^{G}(P(\varphi(h) k \varphi(x) y))-P(\varphi(x) y)\right|\right\|_{l^{\infty}(G)} \\
&= \sup _{\varphi(q) p}\left(\sum_{\varphi(x) y \in G} \mid P(\varphi(h x) A(x) y)(\varphi(h q) A(q) p)\right. \\
&-P(\varphi(x) y)(\varphi(q) p) \mid) \\
&= \sup _{\varphi(q) p}\left(\sum_{\varphi(x) y \in G} \mid T(h x)(h q) S(A(x) y)\left(A(x) \varphi(x)^{-1} \varphi(q) p \varphi\left(q^{-1} x\right)\right)\right. \\
&\left.-T(x)(q) S(y)\left(\varphi(x)^{-1} \varphi(q) p \varphi\left(q^{-1} x\right)\right) \mid\right) \\
& \leq \sup _{\varphi(q) p}\left(\sum_{x \in h^{-1} F} T(h x)(h q)\left(\sum_{y \in K}|S(A(x) y)(A(x) l)-S(y)(l)|\right)\right. \\
&\left.\quad+\sum_{x \in H}|T(h x)(h q)-T(x)(q)|\left(\sum_{y \in K} S(y)(l)\right)\right),
\end{aligned}
$$

where $l=\varphi(x)^{-1} \varphi(q) p \varphi\left(q^{-1} x\right)$ in the last lines of the inequality. The inequality above implies the lemma.

Corollary 5.12 Assume $1 \rightarrow K \rightarrow G \stackrel{\pi}{\rightarrow} H \rightarrow 1$ is exact with $K$ a finite group and $H$ admitting an amenable action. If $\gamma \in A u t(G)$ is an automorphism 
such that $\gamma(K)=K$ then $h a(\gamma)=h a(\dot{\gamma})$ where $\dot{\gamma} \in A$ Aut $(H)$ is the induced automorphism.

Proof. This is an easy consequence of Proposition 5.8 and the previous lemma.

Question 5.13 In the previous corollary if we had required that $H$ be finite (rather than $K)$ would we then get $h a(\gamma)=h a\left(\left.\gamma\right|_{K}\right)$ ? We are only able to solve this problem in the case that $K$ is a finitely generated abelian group (cf. Theorem $6.4)$.

We now deduce the group analogues of [Br, Thm. 3.5] and [BC, Thm. 3.3,3.4]. In particular, this gives an affirmative answer to the analogue of [St, Problem $4.2]$.

Proposition 5.14 Let $K, H$ be groups admitting amenable actions and $\rho$ : $H \rightarrow A u t(K)$ be an action of $H$ on $K$. If $h$ is in the center of $H$ then $h a\left(\rho_{h}\right)=h a(\mathrm{Ad} h)$, where $\mathrm{Ad} h$ is the canonical inner automorphism of $K \rtimes_{\rho} H$ implemented by $h$.

Proof. Write $K \rtimes_{\rho} H=K \times H$ as sets with multiplication $\left(k_{1}, h_{1}\right)\left(k_{2}, h_{2}\right)=$ $\left(k_{1} \rho_{h_{1}}\left(k_{2}\right), h_{1} h_{2}\right)$. Let $\omega=\left\{\left(\rho_{h_{1}}\left(k_{1}\right), h_{1}\right)^{-1}, \ldots,\left(\rho_{h_{m}}\left(k_{m}\right), h_{m}\right)^{-1}\right\}$ be an arbitrary finite set. We also let $\pi: K \rtimes_{\rho} H \rightarrow H$ be the canonical quotient map. Note that for all $n \in \mathbb{N}, \pi(\omega)=\pi\left(\omega \cup \ldots \cup \operatorname{Ad} h^{n}(\omega)\right)$ since $h$ is central.

Now choose $T \in l^{1}\left(H, l^{\infty}(H)\right)$ minimal for $(\pi(\omega), \delta)$, let $F=\operatorname{supp}(T)$ and consider the set

$$
\tilde{\omega}=\bigcup_{i=1}^{m} \bigcup_{x \in h_{i}^{-1} F}\left(1_{K}, x\right)^{-1}\left(k_{i}, 1_{H}\right)^{-1}\left(1_{K}, x\right) .
$$

Using again the fact that $h$ is central, it follows from Lemma 5.11 that $r a(\omega \cup$ $\left.\ldots \cup \operatorname{Ad} h^{n}(\omega), 2 \delta\right)$ is bounded above by

$$
r a(\pi(\omega), \delta) r a\left(\tilde{\omega} \cup \ldots \cup \rho_{h}^{n}(\tilde{\omega}), \delta\right),
$$

for all $n \in \mathbb{N}$.

Hence we deduce that $h a(\operatorname{Ad} h, \omega, 2 \delta) \leq h a\left(\rho_{h}, \tilde{\omega}, \delta\right)$, which implies that $h a(\operatorname{Ad} h) \leq h a\left(\rho_{h}\right)$. The opposite inequality follows from monotonicity (Proposition 5.5).

Corollary 5.15 If $\gamma \in A u t(G)$ and $u \in G \rtimes_{\gamma} \mathbb{Z}$ is the element which implements $\gamma$ in the semidirect product then $h a(\gamma)=h a(\mathrm{Ad} u)$. 
Proposition 5.16 For $\gamma_{i} \in \operatorname{Aut}\left(G_{i}\right), i=1,2$, let $\gamma_{1} \times \gamma_{2}$ denote the product automorphism of $G_{1} \times G_{2}$. Then

$$
\max \left\{h a\left(\gamma_{1}\right), h a\left(\gamma_{2}\right)\right\} \leq h a\left(\gamma_{1} \times \gamma_{2}\right) \leq h a\left(\gamma_{1}\right)+h a\left(\gamma_{2}\right) .
$$

Proof. The lower bound follows from monotonicity. The upper bound is a straightforward application of Lemma 5.11.

Question 5.17 In the situation above do we have $h a\left(\gamma_{1} \times \gamma_{2}\right)=h a\left(\gamma_{1}\right)+$ $h a\left(\gamma_{2}\right)$ ? If the groups $G_{1}, G_{2}$ are abelian then this equality holds since this is a well known property of classical topological entropy (cf. Theorem 4.2).

Example 5.18 Let $G=\coprod_{\mathbb{Z}} G_{0}$ be the restricted direct product of a finite group $G_{0}$ and let $\gamma \in \operatorname{Aut}(G)$ be the shift automorphism (induced by the mapping $i \mapsto i+1$ of $\mathbb{Z})$. It follows from Proposition 3.3 that $\log \left(\operatorname{rank}\left(C_{r}^{*}\left(G_{0}\right)\right)\right) \leq h a(\gamma)$, where $\operatorname{rank}\left(M_{n_{1}}(\mathbb{C}) \oplus \cdots \oplus M_{n_{k}}(\mathbb{C})\right)=n_{1}+\cdots+n_{k}$, while the upper bound $h a(\gamma) \leq \log \left(\left|G_{0}\right|\right)$ is readily seen. It would be interesting to know if we actually have equality at either the upper or lower bound. The difficulty appears to be the same as that encountered in Question 5.17.

It is known that if $G_{1}, G_{2}$ admit amenable actions then the free product $G_{1} * G_{2}$ (without amalgamation) also admits such an action (J.L. Tu - private communication). Using the equivalence with the notion of exactness it follows that the same is true with arbitrary amalgamation as well. If $\gamma_{i} \in A u t\left(G_{i}\right)$ are automorphisms then there is a natural free product automorphism $\gamma_{1} * \gamma_{2} \in$ $\operatorname{Aut}\left(G_{1} * G_{2}\right)$.

Question 5.19 Do we have that $h a\left(\gamma_{1} * \gamma_{2}\right)=\max \left(h a\left(\gamma_{1}\right), h a\left(\gamma_{2}\right)\right)$ ?

Note that if both $G_{1}, G_{2}$ are finite groups then $h a\left(\gamma_{1} * \gamma_{2}\right)=0$ since $\gamma_{1} * \gamma_{2}$ is of finite order.

\section{Crystallographic Groups}

In this section we will compute the dual entropy of an arbitrary automorphism of an extension of a finitely generated free abelian group by a finite group. However, we first require some elementary preliminary results regarding Følner sets in finitely generated abelian groups. 
Fix $p \in \mathbb{N}$, let $v_{1}, \ldots, v_{p}$ be linearly independant vectors in $\mathbb{R}^{p}$ and let $\chi=\left\{v_{1}, \ldots, v_{p}\right\}$. For each $t \in(0, \infty)$, let $\Gamma_{\chi}(t) \subset \mathbb{R}^{p}$ be the parallelpiped

$$
\left\{\sum_{i=1}^{p} s_{i} v_{i}: s_{i} \in \mathbb{R},\left|s_{i}\right| \leq t, 1 \leq i \leq p\right\} .
$$

For any two sets $H, K$ we let $H \triangle K$ denote the symmetric difference.

Lemma 6.1 For each $\delta>0$ there exists a constant $C=C(\delta)$ with the following property: For any set of linearly independant vectors $\chi=\left\{v_{1}, \ldots, v_{p}\right\}$ such that

$$
\Gamma_{\chi}(1) \supset\left\{h \in \mathbb{R}^{p}: h=\left(h_{1}, \ldots, h_{p}\right),\left|h_{i}\right| \leq 1,1 \leq i \leq p\right\},
$$

(where we are using the canonical basis on the right hand side) and any $x \in$ $\Gamma_{\chi}(1) \cap \mathbb{Z}^{p}$ we have

$$
\frac{\left|\left(x+\Gamma_{\chi}(C) \cap \mathbb{Z}^{p}\right) \triangle\left(\Gamma_{\chi}(C) \cap \mathbb{Z}^{p}\right)\right|}{\left|\Gamma_{\chi}(C) \cap \mathbb{Z}^{p}\right|}<\delta .
$$

Proof. Let $\chi=\left\{v_{1}, \ldots, v_{p}\right\}$ be a basis of $\mathbb{R}^{p}$ with the property stated above and let $C>3$ be chosen so that $\left(\frac{C-2}{C+1}\right)^{p}>1-\delta / 2$.

Letting $Q(1)=\Gamma_{\chi}(1) \cap \mathbb{Z}^{p}$ and $x \in Q(1)$ be arbitrary we have

$$
\frac{|(x+Q(C)) \cap Q(C)|}{|Q(C)|} \geq \frac{|Q(C-1)|}{|Q(C)|},
$$

since $Q(C-1) \subset(x+Q(C)) \cap Q(C)$. However, our hypothesis on the set $\chi$ implies the inequalities $|Q(C)| \leq \operatorname{vol}\left(\Gamma_{\chi}(C+1)\right)$ and $\operatorname{vol}\left(\Gamma_{\chi}(C-2)\right) \leq|Q(C-1)|$, where $\operatorname{vol}(\cdot)$ denotes the usual volume in $\mathbb{R}^{p}$. Hence

$$
\frac{|(x+Q(C)) \cap Q(C)|}{|Q(C)|} \geq\left(\frac{C-2}{C+1}\right)^{p} .
$$

But this implies

$$
\frac{\left|\left(x+\Gamma_{\chi}(C) \cap \mathbb{Z}^{p}\right) \triangle\left(\Gamma_{\chi}(C) \cap \mathbb{Z}^{p}\right)\right|}{\left|\Gamma_{\chi}(C) \cap \mathbb{Z}^{p}\right|} \leq 2\left(1-\left(\frac{C-2}{C+1}\right)^{p}\right)<\delta .
$$

We will need to recall [Vo, Lem. 5.2].

Lemma 6.2 Let $\gamma \in G L(p, \mathbb{R})$ with eigenvalues $\lambda_{1}, \ldots, \lambda_{p}$ and define $\mu_{i}=$ $\max \left\{1,\left|\lambda_{i}\right|\right\}$ for $1 \leq i \leq p$. There exists a basis $\left\{v_{1}, \ldots, v_{p}\right\}$ of $\mathbb{R}^{p}$ with the property that if $\varepsilon>0$ and a finite subset $\sigma \subset \mathbb{R}^{p}$ are given then there exists $n_{0} \in \mathbb{N}$ such that for all $n>n_{0}$ we have

$$
\left\{\gamma^{j}(h): h \in \sigma, 1 \leq j \leq n\right\} \subset\left\{\sum_{i=1}^{p} s_{i}(1+\varepsilon)^{n} \mu_{i}^{n} v_{i}: s_{i} \in \mathbb{R},\left|s_{i}\right| \leq 1,1 \leq i \leq p\right\} .
$$


Assume $G=\mathbb{Z}^{p} \oplus F$ where $F$ is a finite abelian group and let $\sigma \in A u t(G)$. Then $\sigma(F)=F$ and hence there is an induced automorphism $\dot{\sigma} \in \operatorname{Aut}(G / F)=$ $G L(p, \mathbb{Z})$. Let $\lambda_{1}, \ldots, \lambda_{p}$ be the eigenvalues of $\dot{\sigma}$ and $\mu_{i}=\max \left\{1,\left|\lambda_{i}\right|\right\}$ for $1 \leq i \leq p$.

Lemma 6.3 Let $G, \sigma \in \operatorname{Aut}(G)$ and $\mu_{1}, \ldots, \mu_{p}$ be as above. For each finite set $\omega \subset G, \delta>0$ and $\varepsilon>0$ there exists a constant $K=K(\omega, \delta, \dot{\sigma})$ such that

$$
r a\left(\omega+\cdots+\sigma^{n}(\omega), 2 \delta\right) \leq|F|(K n)^{p}(1+\varepsilon)^{n p}\left(\mu_{1} \mu_{2} \cdots \mu_{p}\right)^{n} .
$$

Proof. Letting $\pi: G \rightarrow G / F \cong \mathbb{Z}^{p}$ be the quotient map, we see from Lemma 5.11 that

$$
r a\left(\omega+\cdots+\sigma^{n}(\omega), 2 \delta\right) \leq|F| r a\left(\pi(\omega)+\cdots+\dot{\sigma}^{n}(\pi(\omega)), \delta\right) .
$$

Now choose a basis $\chi=\left\{v_{1}, \ldots, v_{p}\right\}$ of $\mathbb{R}^{p}$ as in Lemma 6.2. Then we can find a constant $K_{1}=K_{1}(\omega, \chi)$ such that for all $n \in \mathbb{N}$ we have the inclusion

$$
\bigcup_{j=0}^{n} \dot{\sigma}^{j}(\pi(\omega)) \subset\left\{\sum_{i=1}^{p} s_{i}(1+\varepsilon)^{n} \mu_{i}^{n} v_{i}: s_{i} \in \mathbb{R},\left|s_{i}\right| \leq K_{1}, 1 \leq i \leq p\right\} .
$$

(The constant $K_{1}$ simply insures that the first few iterates of $\pi(\omega)$ are also contained in the right hand side.) Hence we have

$$
\pi(\omega)+\cdots+\dot{\sigma}^{n}(\pi(\omega)) \subset\left\{\sum_{i=1}^{p} s_{i}(1+\varepsilon)^{n} \mu_{i}^{n} v_{i}: s_{i} \in \mathbb{R},\left|s_{i}\right| \leq n K_{1}, 1 \leq i \leq p\right\},
$$

for all $n \in \mathbb{N}$.

Now let $\chi_{n}=\left\{n K_{1}(1+\varepsilon)^{n} \mu_{i}^{n} v_{i}\right\}_{1 \leq i \leq p}$ and $\Gamma_{\chi_{n}}(\cdot)$ be as in Lemma 6.1. We may assume without loss of generality that each $\chi_{n}$ satisfies the technical requirements of that lemma and hence find a constant $C=C(\delta)$ such that for all $n \in \mathbb{N}$ and any $x \in \Gamma_{\chi_{n}}(1) \cap \mathbb{Z}^{p}$,

$$
\frac{\left|\left(x+\Gamma_{\chi_{n}}(C) \cap \mathbb{Z}^{p}\right) \triangle\left(\Gamma_{\chi_{n}}(C) \cap \mathbb{Z}^{p}\right)\right|}{\left|\Gamma_{\chi_{n}}(C) \cap \mathbb{Z}^{p}\right|}<\delta .
$$

Now defining $T_{n} \in l^{1}\left(\mathbb{Z}^{p}\right)$ to be the characteristic function over $\Gamma_{\chi_{n}}(C) \cap \mathbb{Z}^{p}$ and $S_{n}=\left|\Gamma_{\chi_{n}}(C) \cap \mathbb{Z}^{p}\right|^{-1} T_{n}$ one checks that $\left\|x \cdot S_{n}-\left(S_{n}\right)\right\|<\delta$ for all $x \in$ $\Gamma_{\chi_{n}}(1) \cap \mathbb{Z}^{p}$. In particular this shows $r a\left(\pi(\omega)+\cdots+\dot{\sigma}^{n}(\pi(\omega)), \delta\right) \leq\left|\operatorname{supp}\left(S_{n}\right)\right|$. However, we also have that $\left|\operatorname{supp}\left(S_{n}\right)\right|=\left|\Gamma_{\chi_{n}}(C) \cap \mathbb{Z}^{p}\right| \leq \operatorname{vol}\left(\Gamma_{\chi_{n}}(C+1)\right)=$ $\tilde{C}\left(C K_{1} n\right)^{p}(1+\varepsilon)^{n p}\left(\mu_{1} \mu_{2} \cdots \mu_{p}\right)^{n}$, where $\tilde{C}$ is some constant depending only on $\chi$. Letting $K=\max (1, \tilde{C}) C K_{1}$ concludes the proof of the lemma. 
Assume now that $G$ contains a finitely generated normal abelian subgroup $A$ of finite index. Then $A \cong \mathbb{Z}^{p} \oplus F$ for some finite abelian group $F$ and, as above, any automorphism of $\sigma \in A u t(A)$ will induce an automorphism $\dot{\sigma} \in G L(p, \mathbb{Z})$. Also as above we let $\mu_{j}=\max \left(1,\left|\lambda_{j}\right|\right), 1 \leq j \leq p$, for the eigenvalues $\lambda_{j}$ of $\dot{\sigma}$.

Theorem 6.4 If $G$ is as above and $\gamma \in \operatorname{Aut}(G)$ is such that $\gamma$ restricts to an automorphism $\sigma \in \operatorname{Aut}(A)$ then $h a(\gamma)=h a(\sigma)=h a(\dot{\sigma})=\log \left(\mu_{1} \mu_{2} \cdots \mu_{p}\right)$.

Proof. First note that by duality we have $h a(\dot{\sigma})=\log \left(\mu_{1} \mu_{2} \cdots \mu_{p}\right)$ (cf. Theorem 4.2). So by monotonicity and Corollary 5.12 it suffices to show $\log \left(\mu_{1} \mu_{2} \cdots \mu_{p}\right) \geq$ $h a(\gamma)$. Let $\dot{\gamma}$ denote the induced automorphism of $G / A$. Since $h a\left(\gamma^{k}\right)=k h a(\gamma)$ for all positive integers (Proposition 5.3), it suffices to prove the corresponding inequality for some power of $\gamma$. Hence, replacing $\gamma$ with a suitable power, we further assume that $\dot{\gamma}=i d \in \operatorname{Aut}(G / A)$.

Now choose a unital splitting $\varphi: G / A \rightarrow G$ and let $\theta: G / A \times G / A \rightarrow A$ be the associated cocycle map. For each element $x \in G / A$ let $a_{x} \in A$ be the unique element such that $\gamma(\varphi(x))=\varphi(x) a_{x}$ (since $\dot{\gamma}$ is the identity). Note that for all integers $j$ we have $\gamma^{j}(\varphi(x))=\varphi(x) a_{x} \cdots \gamma^{j-2}\left(a_{x}\right) \gamma^{j-1}\left(a_{x}\right)$. Hence if $\omega=\left\{\left(\varphi\left(x_{1}\right) a_{1}\right)^{-1}, \ldots,\left(\varphi\left(x_{m}\right) a_{m}\right)^{-1}\right\} \subset G$ is an arbitrary finite set and we define $\chi_{1}=\left\{a_{1}, \ldots, a_{m}\right\} \cup\left\{a_{x}: x \in G / A\right\} \subset A$ then Lemma 5.11 implies that $r a\left(\omega \cup \ldots \cup \gamma^{n}(\omega), 2 \delta\right)$ is bounded above by

$$
|G / A| r a\left(\left(\bigcup_{x \in G / A} \varphi(x)^{-1}\left(\chi_{1}+\gamma\left(\chi_{1}\right)+\cdots+\gamma^{n}\left(\chi_{1}\right)\right) \varphi(x)\right)+\left(\bigcup_{s, t \in G / A} \theta(s, t)\right), \delta\right),
$$

for all $n \in \mathbb{N}$.

Using the fact that convolution is abelian when the underlying group is abelian, one checks that $r a(X+Y, \delta) \leq r a(X, \delta / 2) r a(Y, \delta / 2)$ for all finite subsets $X, Y \subset A$. So letting $\left.C=|G / A| r a\left(\cup_{s, t \in G / A} \theta(s, t)\right), \delta / 2\right)$ we have the inequality

$r a\left(\omega \cup \ldots \cup \gamma^{n}(\omega), 2 \delta\right) \leq C r a\left(\bigcup_{x \in G / A} \varphi(x)^{-1}\left(\chi_{1}+\gamma\left(\chi_{1}\right)+\cdots+\gamma^{n}\left(\chi_{1}\right)\right) \varphi(x), \delta / 2\right)$,

for all $n \in \mathbb{N}$. Finally, define $\chi_{2}=\cup_{x \in G / A} \varphi(x)^{-1} \chi_{1} \varphi(x) \subset A$ and we have

$$
r a\left(\omega \cup \ldots \cup \gamma^{n}(\omega), 2 \delta\right) \leq C r a\left(\chi_{2}+\ldots+\gamma^{n}\left(\chi_{2}\right), \delta / 2\right),
$$

since $\cup_{x \in G / A} \varphi(x)^{-1}\left(\gamma^{j}(X)\right) \varphi(x)=\gamma^{j}\left(\cup_{x \in G / A} \varphi(x)^{-1} X \varphi(x)\right)$ for all finite subsets $X \subset A$ and $j \in \mathbb{N}$ (this uses that $A$ is abelian).

The previous lemma now states that for every $\varepsilon>0$ we can find some constant $K$ such that

$$
r a\left(\chi_{2}+\ldots+\gamma^{n}\left(\chi_{2}\right), \delta / 2\right) \leq K n^{p}(1+\varepsilon)^{n p}\left(\mu_{1} \mu_{2} \cdots \mu_{p}\right)^{n},
$$


for all $n \in \mathbb{N}$ and hence

$$
\begin{aligned}
h a(\gamma, \omega, 2 \delta) & =\limsup _{n \rightarrow \infty} \frac{\log \left(r a\left(\omega \cup \ldots \cup \gamma^{n}(\omega), 2 \delta\right)\right)}{n+1} \\
& \leq \limsup _{n \rightarrow \infty} \frac{\log \left(C K n^{p}(1+\varepsilon)^{n p}\left(\mu_{1} \mu_{2} \cdots \mu_{p}\right)^{n}\right)}{n+1} \\
& =\log (1+\varepsilon)^{p}+\log \left(\mu_{1} \mu_{2} \cdots \mu_{p}\right) .
\end{aligned}
$$

But since $\varepsilon$ is arbitrary, this proves the theorem.

Remark 6.5 A proof similar to that given above shows that if $1 \rightarrow A \rightarrow G \rightarrow$ $H \rightarrow 1$ is exact with $A$ finitely generated and abelian then for each $\gamma \in A u t(G)$ such that $\gamma(A)=A$ and $\dot{\gamma} \in A u t(H)$ is the identity we have $h a(\gamma)=h a\left(\left.\gamma\right|_{A}\right)$. In particular, if $H$ is also abelian then the dual entropy of all inner automorphisms of $G$ is determined by the restrictions to $A$. (Compare with Corollary 5.15.)

If $H \subset G$ is a subgroup then we let $S_{H}=\{g \in G: g h=h g$ for all $h \in H\}$ denote the stabilizer of $H$ and $Z(H)$ denote the center.

Lemma 6.6 Assume $A \subset G$ is a normal torsion free abelian subgroup of finite index. Then every automorphism of $G$ leaves $Z\left(S_{A}\right)$ invariant (i.e. $\gamma\left(Z\left(S_{A}\right)\right)=$ $Z\left(S_{A}\right)$ for all $\left.\gamma \in \operatorname{Aut}(G)\right)$.

Proof. Set $d=|G / A|$ and consider the set $G^{d}=\left\{g^{d}: g \in G\right\}$. Note that $G^{d} \subset$ $A$ and if $\gamma \in \operatorname{Aut}(G)$ then $\gamma\left(G^{d}\right)=G^{d}$. Thus if $g \in \gamma\left(S_{A}\right)$ then $g$ commutes with $a^{d}$ for all $a \in A$ since $a^{d} \in G^{d}=\gamma\left(G^{d}\right) \subset \gamma(A)$. Thus $a^{d}=g a^{d} g^{-1}=\left(g a g^{-1}\right)^{d}$. But, since $A$ is abelian, this implies $1_{g}=\left(a^{-1} g_{a g}{ }^{-1}\right)^{d}$ which implies $g a=a g$ since $A$ is torsion free. Thus $\gamma\left(S_{A}\right) \subset S_{A}$ and the same argument applied to $\gamma^{-1}$ implies $\gamma\left(S_{A}\right)=S_{A}$. But if $\gamma$ leaves $S_{A}$ invariant then it also leaves $Z\left(S_{A}\right)$ invariant.

We are finally in a position to compute $h a(\cdot)$ for all automorphisms of crystallographic groups. First, assume that $1 \rightarrow \mathbb{Z}^{p} \rightarrow G \rightarrow F \rightarrow 1$ is exact, with $F$ a finite group. Let $A=Z\left(S_{\mathbb{Z}^{p}}\right)$. Then $A$ is a finitely generated normal abelian subgroup of $G$ of finite index. Writing $A \cong \mathbb{Z}^{q} \oplus L$ for some $q \in \mathbb{N}$ and finite abelian group $L$ we can define a homomorphism $\rho: \operatorname{Aut}(G) \rightarrow G L(q, \mathbb{Z})$ in the following manner. If $\gamma \in \operatorname{Aut}(G)$ then the previous lemma says that $\gamma$ leaves $A$ invariant and hence $\left.\gamma\right|_{A}$ leaves $L$ invariant and hence defines an element $\rho(\gamma) \in \operatorname{Aut}(A / L)=G L(q, \mathbb{Z})$. As a consequence of Theorem 6.4 we get:

Corollary 6.7 Let $G$ and $\rho: \operatorname{Aut}(G) \rightarrow G L(q, \mathbb{Z})$ be as above. Then for every $\gamma \in \operatorname{Aut}(G)$,

$$
h a(\gamma)=h a(\rho(\gamma))=\log \left(\mu_{1} \mu_{2} \cdots \mu_{q}\right),
$$

where $\mu_{j}=\max \left(1,\left|\lambda_{j}\right|\right), 1 \leq j \leq q$, for each eigenvalue $\lambda_{j}$ of $\rho(\gamma)$. 
Acknowledgements. The first named author would like to thank his thesis advisor, Marius Dadarlat, for a number of important discussions related to entropy in (amenable) groups. Indeed, a number of the ideas presented here can be traced back to those conversations.

The present collaboration was made possible by the authors' participation in the program Probabilités Libres et Espaces D'Opérateurs, held at Institut Henri Poincaré. We wish to thank the institute for it's support and hospitality and the organizers for putting together such a stimulating semester.

\section{References}

[Ad] S. Adams, Boundary amenability for word hyperbolic groups and an application to smooth dynamics of simple groups, Topology 33 (1994), 765 783.

[AD] C. Anantharaman-Delaroche, Amenability and exactness for dynamical systems and their $C^{*}$-algebras, preprint.

[ADR] C. Anantharaman-Delaroche and J. Renault, Amenable groupoids, to appear in L'Enseignement Mathémathique

[Br] N.P. Brown, Topological entropy in exact $C^{*}$-algebras, Math. Ann. 314 (1999), $347-367$.

[BC] N.P. Brown and M. Choda, Approximation entropies in crossed products with an application to free shifts, preprint.

[Ch] M. Choda, Entropy on crossed products and entropy on free products, preprint.

[CNT] A. Connes, H. Narnhofer and W. Thirring, Dynamical entropy of $\mathrm{C}^{*}$ algebras and von Neumann algebras, Comm. Math. Phys. 112 (1987), 691 - 719 .

[CS] A. Connes and E. Størmer, Entropy of $I I_{1}$ von Neumann algebras, Acta Math. 134 (1975), 289 - 306.

[Dy] K. Dykema, Topological entropy for some automorphisms of reduced amalgamated free product $C^{*}$-algebras, preprint.

[Ge] Emmanuel Germain, Approximate invariant means for boundary actions of hyperbolic groups, appendix to [ADR].

[GK] E. Guentner and J. Kaminker, Exactness and the Novikov Conjecture, preprint. 
[HR] N. Higson, J. Roe Amenable group actions and the Novikov Conjecture Preprint 1999

[O] N. Ozawa, Amenable actions and exactness for discrete groups, preprint.

[Pa] V. Paulsen, Completely bounded maps and dilations, Pitman Research Notes in Mathematics, vol. 146, Longman, 1986.

[Pe] G. Pedersen, $C^{*}$-algebras and their automorphism groups, Academic Press, London, 1979.

[Pet] J. Peters, Entropy on discrete abelian groups, Adv. Math. 33 (1979), 1 13.

[SS] A.M. Sinclair and R.R. Smith, The completely bounded approximation property for discrete crossed products, Indiana Univ. Math. J. 46 (1997), 1311 $-1322$.

[St] E. Størmer, Entropy in operator algebras, Asterisque 232 (1995), 211 - 230.

[Vo] D. Voiculescu, Dynamical approximation entropies and topological entropy in operator algebras, Comm. Math. Phys. 170 (1995), 249 - 281.

[Wal] P. Walters, An introduction to ergodic theory, Grad. Texts Math. 79, Springer-Verlag, New York-Berlin (1982).

[Z] R.J. Zimmer Ergodic theory and semi-simple Lie groups Birkhäuser, 1984. 